\title{
Upper Extremity Rehabilitation of Children with Cerebral Palsy using Accelerometer Feedback on a Multitouch Display
}

\author{
Alan Dunne, Son Do-Lenh, Gearóid Ó' Laighin, Chia Shen, Paolo Bonato
}

\begin{abstract}
Cerebral palsy is a non-progressive neurological disorder caused by disturbances to the developing brain. Physical and occupational therapy, if started at a young age, can help minimizing complications such as joint contractures, and can improve limb range of motion and coordination. While current forms of therapy for children with cerebral palsy are effective in minimizing symptoms, many children find them boring or repetitive. We have designed a system for use in upper-extremity rehabilitation sessions, making use of a multitouch display. The system allows children to be engaged in interactive gaming scenarios, while intensively performing desired exercises. It supports games which require completion of specific stretching or coordination exercises using one or both hands, as well as games which use physical, or "tangible" input mechanisms. To encourage correct posture during therapeutic exercises, we use a wireless kinematic sensor, worn on the patient's trunk, as a feedback channel for the games. The system went through several phases of design, incorporating input from observations of therapy and clinical sessions, as well as feedback from medical professionals. This paper describes the hardware platform, presents the design objectives derived from our iterative design phases and meetings with clinical personnel, discusses our current game designs and identifies areas of future work.
\end{abstract}

\section{INTRODUCTION}

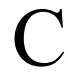
erebral palsy describes a group of permanent physical disorders caused by disturbances to the fetal or infant brain. This disorder presents as a motor dysfunction characterized by weakness, spasticity or other motor dysfunction, affecting one or more limbs. Motor impairment is often

This work was supported in part by the Irish Council for Science, Engineering and Technology under the Enterprise Partnership Scheme.

Alan Dunne is with the Bioelectronics Research Cluster, National Centre for Biomedical Engineering Science at the National University of Ireland, Galway (email: a.dunne13@nuigalway.ie)

Son Do-Lenh was with the SDR Lab, School of Engineering and Applied Sciences, Harvard University. He is now with CRAFT, Swiss Federal Institute of Technology Lausanne (EPFL), 1015 Lausanne, Switzerland (email: son.dolenh@epfl.ch)

Gearóid Ó'Laighin is with the School of Engineering \& Informatics and the Bioelectronics Research Cluster, National Centre for Biomedical Engineering Science, NUI Galway, University Road, Galway, Ireland (email: gearoid.olaighin@nuigalway.ie)

Chia Shen, is with the School of Engineering and Applied Sciences, Harvard University, Cambridge, MA 02138 USA. (e-mail: chia_shen@harvard.edu)

Paolo Bonato is with the Dept. of Physical Medicine and Rehabilitation, Harvard Medical School, Spaulding Rehabilitation Hospital, Boston, MA (corresponding author, phone: 617-573-2770; fax: 617-5732769; e-mail: pbonato@partners.org). accompanied by other symptoms such as a disturbance in cognition or communication, vision or auditory impairment or intellectual deficit. The incidence of cerebral palsy is approximately 2 per 1000 live births, with the rate increasing in babies born prematurely or severely underweight $[1,2]$.

While cerebral palsy is non-progressive, meaning it will neither improve nor worsen over time, it is possible to manage the symptoms to some degree through occupational and physical therapies. Upper extremity impairments shown by children with cerebral palsy are marked by limited range of movement at the shoulder (which shows exaggerated adduction and internal rotation) and the elbow (showing limited extension). Also, children with cerebral palsy often show limited control of the forearm (especially in performing supination movements), and of the wrist and hand movements (often observed as limited extension of the fingers and thumb abduction and extension) [3]. Surgery is an option to address certain impairments, but is generally a last resort, particularly before the patient has fully grown.

Therapy for children with cerebral palsy can improve muscle strength, muscle endurance and overall joint range of motion. A therapy program involving many low resistance repetitions of a particular exercise will enhance local muscular endurance, and passive, static, gentle stretches are performed on individual joints in order to decrease and prevent severe joint contractures [4]. Occupational therapy has been shown to be effective in improving and maintaining fine motor abilities in children with cerebral palsy [4]. A large challenge in administering therapy, however, is to maintain the child's interest and enthusiasm during these exercises. In this paper, we present a new system which aims to support the design of therapeutic exercises used in upper limb rehabilitation of children with cerebral palsy by integrating an augmented interactive tabletop, physically graspable objects (tangibles) and wearable accelerometer technologies.

\section{RELATED WORK}

Several systems for rehabilitation using multitouch technology have been designed to date, however none have been designed specifically for patients with cerebral palsy. Leitner et al. [5] investigated the potential of a tabletop device combined with tangible inputs in a rehabilitation setting, and described their design of three concepts for rehabilitation exercises. The games concentrated on the area 
of visual impairment and visual perception symptoms.

Mumford et al. [6] describe a virtual tabletop workspace for upper-limb rehabilitation, and evaluated its effectiveness in promoting movement skills in patients with traumatic brain injury [7]. Participants demonstrated improvements in movement accuracy, efficiency and bimanual dexterity. The system uses a large monitor placed horizontally, augmented with feedback from a stereo camera system.

Annett et al. [8] describe the design of a multitouch tabletop for upper-extremity motor rehabilitation, the AIR Touch. The system comprised several games and put emphasis on designing games requiring performance of repeatable, measurable tasks. The games were for general upper-limb rehabilitation, and did not focus on one particular type of impairment or functional limitation.

None of these projects have used wearable accelerometers to track and discourage compensatory movement strategies during therapy. Much work has been done with kinematic sensors in a medical context, and they have previously been used to evaluate the severity of spasticity in children with cerebral palsy [9]. In this paper, a system was designed to measure the three-dimensional orientation of limbs during motion, and algorithms were developed relying on the estimated orientation of limbs to discourage compensatory movement strategies during therapy.

\section{HARDWARE AND SOFTWARE PLATFORM}

Our system (Fig. 1) consists of three primary hardware components: a multitouch display, tangible object inputs, and a wearable accelerometer to sense movement and rotation, used for game scoring feedback. We use a Microsoft Surface interactive multitouch display. The Surface uses five internal cameras to measure reflections from infrared light on its 30 " display. It is capable of simultaneously recognizing physical objects and finger touch input, as well as specially designed fiducials or "tags".

Using the Bluetooth protocol, the Surface platform is connected to a small accelerometer, attached to a custommade vest worn by the patient. This is used to measure trunk rotation. The accelerometer data is sampled at $100 \mathrm{~Hz}$ and transmitted to the Surface, which processes the data and uses this feedback in the games. The metric used is trunk flexion, which indicates a patient leaning forward to compensate for limited range of movement in the elbow and/or shoulder. When a patient leans forward past a certain threshold, an event is triggered in the game. An onscreen indicator shows the patient's current body position in relation to this threshold, allowing for self-correction when this point is being approached. The threshold level for trunk rotation for each patient is different, and can be adjusted by the therapist so as to match each rehabilitation exercise to the patient's ability. In this way, the therapist can make the exercises more challenging as the patient's range of motion improves over time. The software was developed in C\# and XAML, using the Microsoft Surface software development kit.

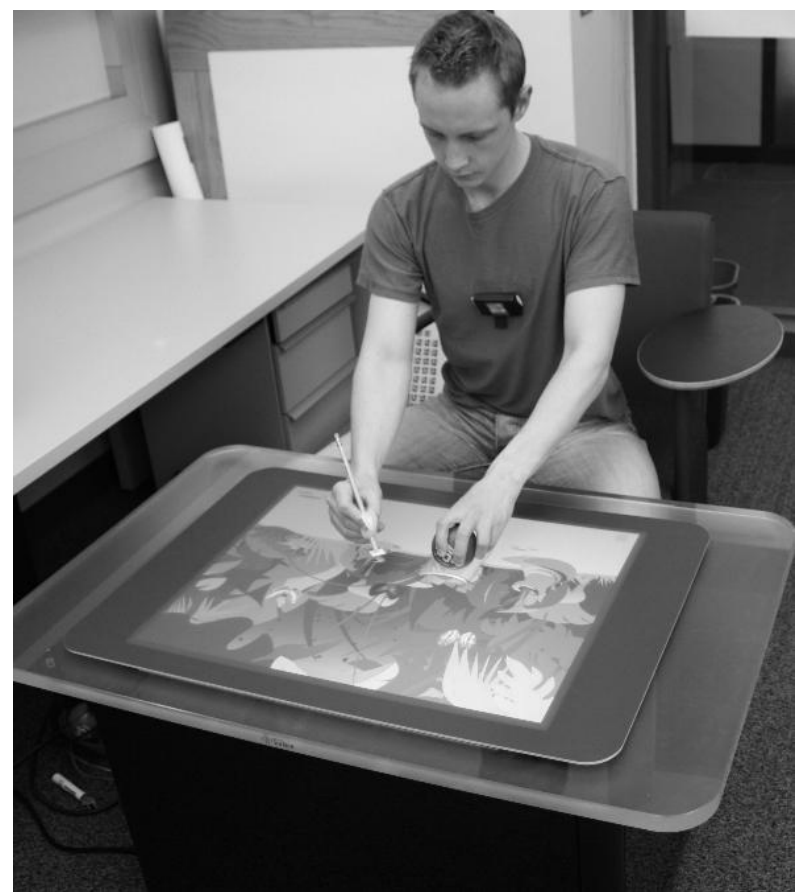

Fig. 1. The complete system in use. The user is wearing the accelerometer and using tangible inputs while playing the "Butterfly" game.

\section{Design ObJectives}

Our aim was to design a system to be used by occupational therapists in therapy sessions with children with cerebral palsy seven to eleven years of age, or cognitive equivalent demographics of pediatric patients.

Three iterative steps were taken in the design process. First, we analyzed current literature in order to identify which types of therapeutic interventions would be suitable for use with our system. Secondly, the authors attended cerebral palsy clinics and therapy sessions at Spaulding Rehabilitation Hospital, Boston, and Boston Children's Hospital. And thirdly, the system went through several phases of redesign based on feedback from clinicians who used the system.

One of the most important features of this system is the ability to track patient trunk position and discourage compensatory movement strategies during therapy sessions. In everyday life, patients with impaired movement of the upper limbs often use their trunk movement to compensate for the lack of extension of upper limb joints, for example to make it easier to reach for objects. If these compensatory movements are made during therapy, they would have a negative impact on the efficacy of the rehabilitation exercises children are required to perform. By monitoring movements of the trunk, these movements can be detected and discouraged using feedback within the games, in order to maximize the effectiveness of therapy sessions.

Another feature of our system is to provide an immersive and engaging environment, while providing a platform to perform therapeutic exercises. This is a key point, as it has 
been shown that immersion in a virtual environment can help reducing the amount of pain experienced by both adult [10] and pediatric [11,12] patients during rehabilitation. By designing the system to encourage completion of therapy activities to meet in-game goals, therapy sessions are made intrinsically rewarding to the patient.

The system has been designed to use tangible objects as input mechanisms. Several "wands" of different diameters are used with a set of custom grips of varying size, shape and contour, commonly used by children with cerebral palsy. These are modified with 8-bit tag patterns which are recognized by the multitouch display (Fig. 2). Using tangible objects also allows us to combine reaching exercises with grasp and release motor tasks, using different types of grips for fine motor exercises. For example, a foam ball is used as an input device for some of our games. This provides tangible resistance to counteract involuntary finger and thumb flexion in a patient's hand -such as the weaker hand of a patient with hemiparesis. The games encompass the performance of both unimanual and bimanual tasks, as recent research [13-15] reveals the effectiveness of this combination for rehabilitation.

It was necessary to design the games so that they may be used by patients with a broad range of ability - in terms of both motor ability and cognitive function.

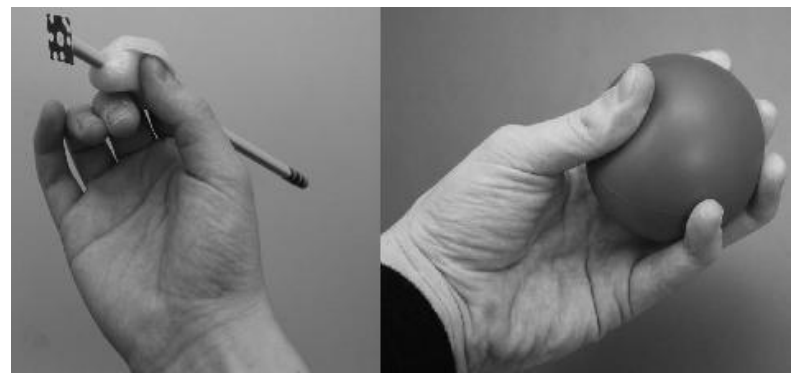

Fig. 2. Examples of the tangible interaction objects: a pencil modified with an optical tag and a customized grip on the left, and a foam ball used to counteract involuntary finger flexion on the right.

\section{REHABILITATION GAMES}

While our hardware and software platforms support the development of many types of games involving both touch and tangible inputs, we only present here typical games that demonstrate important representative features.

\section{A. Find the bone}

This is a simple game in which the child needs to maneuver a bone to the dog using their finger, avoiding the other characters and the obstacles in the game landscape. Points are scored by collecting stars, which are placed randomly onscreen using constraints set by the therapist. More points are awarded if the patient is in an upright position. Using tangible objects, the therapist can choose the location of the hedges which the patient needs to avoid, in order to tailor the game to each child's motor ability. A less complex version of this game - called "Maze Tracing" - requires the patient to draw with their finger within the winding tunnel of a maze. "Find the bone" embodies some of the "Maze Tracing" exercises while providing the child with more flexibility and the therapist with more adaptability.

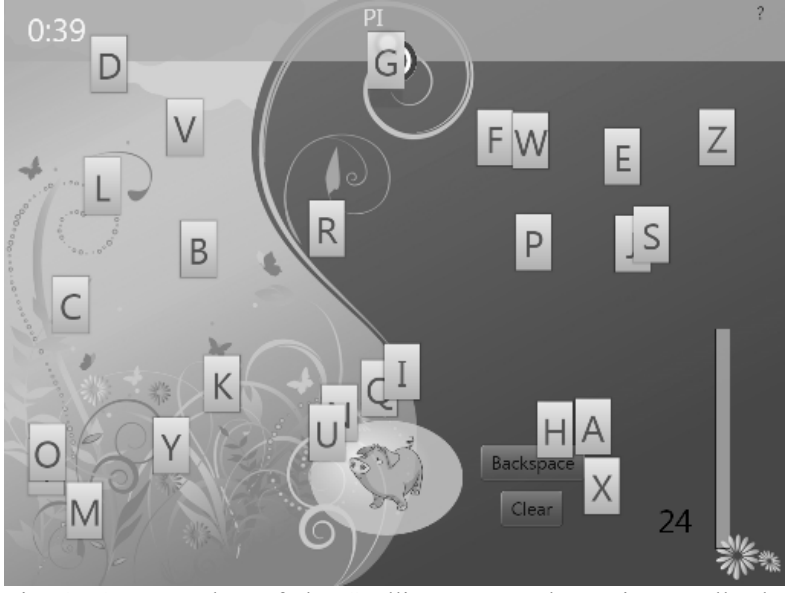

Fig. 3. A screenshot of the Spelling game. The patient spells the animal shown in the bubble, while the timer in the top left counts down. Their score is shown in the bottom right. The letter panel at the top shows the word currently being spelled.

\section{B. Spelling}

In this game (Fig. 3), the patient must spell the animal shown in the bubble onscreen using the letter tiles scattered on the playing surface. Points are awarded depending on how quickly the words are correctly spelled, and how many animals are spelled within a certain time limit. A scoreboard displayed after the game encourages the child to beat their previous scores and allows progress to be tracked over time. Two different methods of interaction may be used: 1) the letters can be selected by tapping them with the "Wand" input tool, or 2) the tiles can be thrown at the onscreen target using a toss gesture. If the child's compensatory movement exceeds a limit set for the accelerometer by the therapist, the tiles will scatter around the display.

\section{Catch the butterflies}

This game (Fig. 4) is designed bearing particular attention to rehabilitation methods for patients with hemiparesis weakening or paralysis in one side of the body. The game uses bimanual input, and requires co-ordination of both hands in order to capture the butterflies in the virtual jar. The jar must be positioned just below the moving butterflies using the foam ball in order for them to be captured, by touching them with the specially tagged "wand" input tool. As the ball is controlled by the patient's weaker hand, the game is designed such that it does not need to be positioned as far from the patient as the wand, which will generally be controlled by the stronger hand. If the patient leans forward too much, the wearable accelerometer sensor will produce a trigger and the butterflies will fly away, and if the ball leaves contact with the screen for too long, the captured butterflies will escape from the jar. Therapists can modify 
the game parameters during the session to suit each patient, for example by changing the sensitivity of the accelerometer or the speed and location of the moving butterflies.

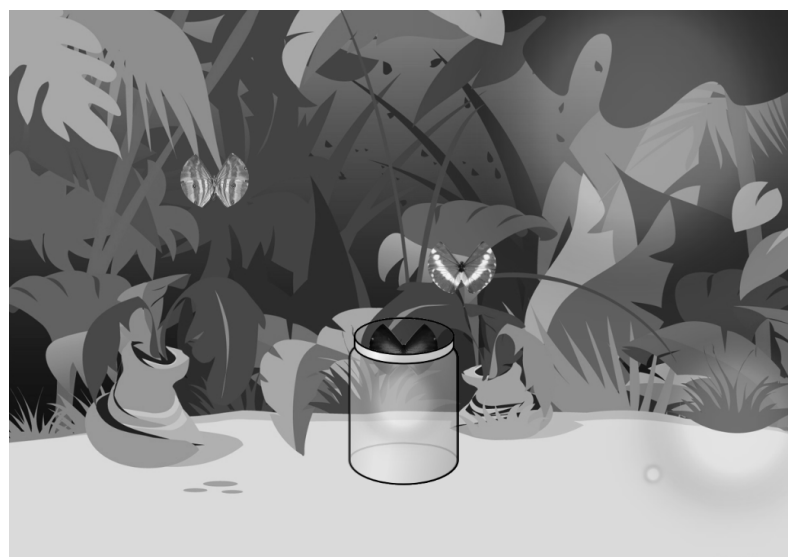

Fig. 4. A screenshot from "Catch the butterflies," a game requiring bimanual interaction. Both of the tangibles shown in Fig. 2 are used, and both dominant and non-dominant hand must be coordinated in order to catch the butterflies in the jar.

\section{CONCLUSIONS AND FUTURE WORK:}

During the design of our system, we interacted with clinicians and solicited their feedback. Their comments and suggestions were used to ensure our system was both appropriate for use with our target population and easy to use by therapists. More input mechanisms are being investigated which will mirror and enhance exercises currently used in therapy. For example, a common exercise is a squeezing action. This exercise helps improving strength, but it is difficult to motivate patients to perform this action. Interactive games could help achieving this goal.

Our current hardware does not directly measure range of motion, but rather the patient's compensatory activities. Recent research has used wearable technology to estimate clinical scores of functional level of stroke patients [16]. The provision of this detail would be a useful addition to our system, as it would provide a more quantitative measure of a patient's ability and progress over time.

More complex combinations of accelerometer feedback and touchscreen data are also being researched, to allow for better quantification of range of motion such as counting the number of correct bimanual stretches performed. This data would allow therapists to track a patient's progress, and combined with the immersiveness of a virtual environment, would motivate patients to perform better in therapy sessions.

One observation by therapists was that in some cases a certain amount of trunk flexion is unavoidable - for example slow, lateral leaning in patients with hemiplegia. This will be integrated into further revisions of the system. Another common suggestion from therapists was to tilt the display at an angle towards the patient. This would enable threedimensional reaching tasks to be used with patients, and allow further fine tuning of the system to suit patients' individual needs.

\section{ACKNOWLEDGEMENT}

The authors would like to thank Ulrike Berzau, Michelle Coldwell, Debra Margolis, Dr. Donna Nimec, and Dr. Brian Snyder for their valuable insights and feedback.

\section{REFERENCES}

[1] P. Rosenbaum, N. Paneth, A. Leviton, M. Goldstein, M. Bax, "A report: the definition and classification of cerebral palsy", Developmental Medicine \& Child Neurology, 49: 8-14, 2006.

[2] A. Johnson, "Prevalence and characteristics of children with cerebral palsy in Europe," Developmental Medicine \& Child Neurology, 44: 633-640, 2002.

[3] M. A. Tonkin, "The upper limb in cerebral palsy," Current Orthopaedics, 9: 149-155, 1995.

[4] D. R. Patel, "Therapeutic Interventions in Cerebral Palsy," Indian Journal of Pediatrics, 72(11): 979-983, 1995.

[5] M. Leitner, M. Tomitsch, T. Költringer, K. Kappel, T. Greshenig, "Designing tangible tabletop interfaces for patients in rehabilitation," in Conference \& Workshop on Assistive Technologies for People with Vision \& Hearing Impairments: Assistive Technology for All Ages (CVHI 2007), M. A. Hersh, Ed. Spain Aug. 2007

[6] N. Mumford, J. Duckworth, R. Eldridge, M. Guglielmetti, P. Thomas, D. Shum, H. Rudolph, G. Williams, P. H. Wilson, "A virtual tabletop workspace form upper-limb rehabilitation in traumatic brain injury (TBI): A multiple case study evaluation," in Virtual Rehabilitation, Vancouver, Canada, 175-180, 2008.

[7] N. Mumford, J. Duckworth, P. R. Thomas, D. Shum, G. Williams, P. H. Wilson, "Upper limb virtual rehabilitation for traumatic brain injury: Initial evaluation of the elements system," Brain Injury, 24(5): 780-791, 2010.

[8] M. Annett, F. Anderson, D. Goertzen, J. Halton, Q. Ranson, W. F. Bischof, P. Boulanger, "Using a Multi-touch Tabletop for UpperExtremity Motor Rehabilitation," in Proc. $21^{\text {st }}$ Annual Conference of the Australian Computer-Human Interaction Special Interest Group: Design: Open 24/7, Melbourne, Australia, 261-264, 2009.

[9] J. C. van den Noort, V. A. Scholtes, J. Harlaar, "Evaluation of clinical spasticity assessment in Cerebral palsy using inertial sensors," Gait \& Posture, 30: 138-143, 2009.

[10] H. G. Hoffman, D. Patterson, G. Carrougher, "Use of Virtual reality for Adjunctive Treatment of Adult Burn Pain During Physical Therapy: A Controlled Study," The Clinical Journal of Pain, 16(3): 244-250, 2000.

[11] E. Steele, K. Grimmer, B. Thomas, B. Mulley, I. Fulton, H. Hoffman, "Virtual Reality as a Pediatric Pain Modulation Technique: A Case Study," CyberPsychology \& Behavior, 6: 633-638, 2003.

[12] D. A. Das, D. A. Grimmer, A. L. Sparnon, S. E. McRae, B. H. Thomas, "The efficacy of playing a virtual reality game in modulating pain for children with acute burn injuries: A randomized controlled trial," BMC Pediatrics, 5, 2005

[13] S. L. Kilbreath, J. Crosbie, C. G. Canning, M. J. Lee, "Inter-limb coordination in bimanual reach-to-grasp following stroke," Disabil Rehabil, 28: 1435-43, 2006.

[14] G. N. Lewis, E. J. Perrault, "Side of lesion influences bilateral activation in chronic, post-stroke hemiparesis," Clin Neurophyisiol, 118: 2050-62, 2007.

[15] L. Sheppard, H. Mudie, E. Froude, “An investigation of bilateral isokinematic training and neurodevelopmental therapy in improving use of the affected hand in children with hemiplegia," Phys Occup Ther Pediatr, 27: 5-25, 2007.

[16] S. Patel, R. Hughes, T. Hester, J. Stein, M. Akay, J. G. Dy, P. Bonato, "A novel approach to monitor rehabilitation outcomes in stroke survivors using wearable technology," Proc IEEE, 98(3): 1-12, 2010. 